\title{
Development of an instrument for the identification of frail older people as a target population for integrated care
}

\begin{abstract}
\section{Background}

Primary care is increasingly interested in the identification of frailty, as it selects the target population for integrated care. However, instruments for the identification of frailty specifically validated for use in primary care are scarce. This study developed the Easycare Two-step Older persons Screening (EasycareTOS), which provides a valid, efficient, and pragmatic screening procedure to identify frail older people.
\end{abstract}

\section{Aim}

This paper aims to describe the development of the Easycare-TOS and the data from the pilot studies.

\section{Design and setting}

Observational pilot study in seven academic GP practices in and around Nijmegen, The Netherlands.

\section{Method}

The Easycare-TOS was developed in a cyclic process with the input of stakeholders. In every cycle, the requirements were first defined, then translated into a prototype that was tested in a pilot study. The Easycare-TOS makes optimal use of prior knowledge of the GP, and the professionals' appraisal is decisive in the frailty decision, instead of a cut-off score. Further, it considers aspects of frailty, as well as aspects of the care context of the patient.

\section{Results}

The pilot data have shown that after step 1 , two-thirds of the patients do not need further assessment, because they are judged as not frail, based on prior knowledge of the GP. The overall prevalence of frailty in this pilot study is $24 \%$. Most professionals who participated in the pilot studies considered the time investment acceptable and the method to be of added value.

\section{Conclusion}

The Easycare-TOS instrument meets the predefined efficiency, flexibility, and acceptability requirements for use as an identification instrument for frailty in primary care

\section{Keywords}

delivery of health care, integrated; efficiency: feasibility studies; frail elderly; primary health care.

\section{INTRODUCTION}

It is well recognised that care for community-dwelling older people needs improvement. ${ }^{1,2}$ Current health and social services are particularly insufficient for frail older people who suffer from multiple health and social problems., ${ }^{3,4}$ Care should change from being reactive and disease oriented to proactive and patient oriented; this is often called integrated care. ${ }^{5,6}$ First, though, the older people who would benefit from integrated care, that is those who are frail, must be identified.?

Many instruments for the identification of frailty can be found in the literature. Some, such as the Fried frailty criteria, focus on frailty as a physical syndrome, 8,9 whereas others use a 'broader' definition in which psychological and social aspects are incorporated, such as the Frailty Index by Rockwood and the Tilburg Frailty Indicator (TFI). ${ }^{10-12}$ This 'broader' definition is more consistent with the way the term frailty is used by clinicians in primary care. ${ }^{12}$ However, most of these instruments are not specifically validated for use in primary care. Furthermore, the existing instruments have other limitations that make them less suitable for use in primary care. First, the available instruments neglect information about patients that is already available, such as prior knowledge of the professionals involved. Yet, it is probably

JAL van Kempen, MD, registrar in geriatric medicine; $\mathbf{A}$ Jacobs, MD, registrar in geriatric medicine; SHM Robben, MD, registrar in geriatric medicine; RJF Melis, MD, PhD, senior registrar in geriatric medicine; MGM Olde Rikkert, MD, PhD, professor in geriatric medicine, Department of Geriatric Medicine, Radboud University Nijmegen Medical Centre, Nijmegen, the Netherlands. HJ Schers, MD, PhD, GP; F Ruikes, MD, GP, Department of Primary and Community Care, Centre for Family Medicine, Geriatric Care and Public Health, Radboud University Nijmegen Medical Centre, Nijmegen, the Netherlands. SU Zuidema, MD, PhD, elderly care physician, Department of General Practice, University of more efficient to make use of the existing infrastructure of primary care, and to profit from the information that GPs and their teams already have of individual patients. In addition, the existing frailty instruments use cut-off scores and neglect tacit knowledge. In clinical decision making, most GPs rely not only on explicit factual knowledge, but also on tacit (implicit) knowledge. ${ }^{13}$ Hence, using the professionals' appraisal in a frailty instrument, in addition to a standardised frailty instrument, might have added value.

A final limitation of the existing instruments is that most of them do not take into account the care context of the patient. As the requirement is to identify frail older people in need of integrated care, it is important to consider this context, since it is strongly related to the frailty status of a patient. ${ }^{14}$ The significance of considering the care context in relation to frailty is that it focuses on relevant contextual factors that may require more integration of the care system.

Taking into account the above-mentioned limitations of existing frailty instruments, this study developed a new frailtyidentification instrument with the input of stakeholders from primary care. This instrument was based on the EASY-Care assessment system, ${ }^{15}$ which is a tool for geriatric assessment in primary care and is a good starting point for integrated care.

Groningen, University Medical Centre Groningen, Groningen, the Netherlands.

\section{Address for correspondence}

Janneke van Kempen, Department of Geriatric Medicine, PO Box 9101, 6500 HB Nijmegen, the Netherlands.

E-mail: J.vanKempendger.umcn.nl Submitted: 21 May 2012; Editor's response: 19 July 2012; final acceptance: 20 September 2012 This is the full-length article (published online 25 Feb 2013) of an abridged version published in print. Cite this article as: $\mathbf{B r} \mathbf{J}$ Gen Pract 2013; DOI: 10.3399/bjgp13X664289 


\section{How this fits in}

In the literature, many instruments for the identification of frailty can be found. However, these instruments have scarcely been validated for use in primary care. Therefore, the Easycare Two-step Older persons Screening (Easycare-TOS) was developed. Distinctive characteristics of the Easycare-TOS are that it makes optimal use of prior knowledge of the GP and that, instead of a cut-off score, the professionals' appraisal is decisive in the judgement of frailty.

This article describes the development process of the Easycare Two-step Older persons Screening (Easycare-TOS), and answers the question as to whether it is possible to develop an efficient, flexible, and acceptable method for the identification of community-dwelling frail older people as a target population for integrated care.

\section{METHOD}

\section{Development and basic requirements of the Easycare-TOS}

The Easycare-TOS was developed in a cyclic process. Every cycle started with the research group defining basic requirements, followed by translation of these into a prototype, which was subsequently tested in a pilot study. The research group consisted of a GP, a clinical geriatrician, a nursing home physician, and a researcher. This group defined, refined, and operationalised the requirements by using the input of stakeholders participating in the pilot studies. Based on a literature study and discussion, the research group started the development process by defining the basic requirements for the identification method. These are listed below.

1. The method should fit the 'broad' definition of frailty. The following definition was used: a frail older person has decreased reserve capacity because of multiple health, mental, or social problems; this makes the person vulnerable to changes in the biopsychosocial context, especially when compensating factors are lacking. ${ }^{8,16-18}$

2. The method should be feasible and efficient. This is operationalised by making best use of physicians' prior knowledge.

3. Frailty should be based on the professionals' appraisal after using a standardised frailty instrument. In this way, the professionals make optimal use of their tacit knowledge in this method.

After requirements were defined, the literature was searched for existing instruments for the identification of frailty. In 2009, De Lepeleire proposed a two-step approach with a simple heuristic tool as the first step, and a more complex assessment as the second. ${ }^{19}$ For efficiency reasons, it was decided to adopt this two-step approach for the identification method in the present study. Several short instruments were suggested in the literature for use as a first step..$^{20}$ However, it was not possible to find a suitable instrument that met the basic requirements for this study. Therefore, a new instrument was developed for the first step.

\section{The Easycare-TOS}

The first step is a short instrument (Box 1) that makes a preselection in older people based on prior knowledge of the GP. The GP reviews the patient record and answers 14 questions about the functioning of the patient in somatic, psychological, and social domains. The 14 questions are meant to trigger the GP into considering all relevant aspects. Subsequently, the GP decides whether: (1) the patient is not frail; (2) the patient is frail; or (3) the existing information on the patient is insufficient for making this decision (unclear). The patients who are judged as 'unclear' or 'frail' in the first step are eligible for the second step. In the patients categorised as 'unclear', the second step is aimed at gaining clarity about the presence or absence of frailty. In the patients categorised as 'frail', the aim of the second step is to collect data on all aspects of functioning that are relevant for the care of a person who is frail. In the second step, additional information is collected through a structured assessment by a primary care nurse. This assessment is based on the EASY-Care assessment system. ${ }^{15}$ Box 2 presents several questions from the questionnaire of the second step. Subsequently, the GP and primary care nurse discuss the information collected through the assessment and make a final frailty decision for the 'unclear' patients. The frailty decision in both steps is based on clinical reasoning, and not a numerical score, and is made - using all available explicit and implicit (tacit) information - by considering which factors make the patient more or less frail.

\section{Pilot study 1}

To determine the feasibility, the first version of the Easycare-TOS was tested in a small 


\section{Box 1. The first step of the Easycare-TOS}

\section{Multimorbidity, patient has:}

- 0 or 1 important chronic diseases

- 2 important chronic diseases

- 3 or more important chronic diseases

- Unknown

2. Polypharmacy, patient has:

- Less than 4 chronic medications

- Four or more chronic medications

- Unknown

3. Cognitive problems, patient has:

- No cognitive problems

- Mild cognitive problems

- Dementia (diagnosed)

- Unknown

4. Hearing and vision, patient has:

- No problems with hearing and vision

- Mild problems with hearing and vision

- Obvious problems with hearing and vision

- Unknown

\section{Activities of daily living, patient is:}

- Not dependent on professional or informal care

- To some extent dependent on professional or informal care

- Highly dependent on professional or informal care

- Unknown

\section{Mobility, patient is:}

- Able to move independently

- Able to move with some help

- Unable to move

- Unknown

7. Falls, patient has:

- Not fallen the past 12 months

- Fallen 1 time in the past 12 months

- Fallen 2 times or more in the past 12 months

- Unknown

8. Informal care, patient has:

- Sufficient informal care

- Insufficient informal care

- No informal care

- Unknown

9. Loneliness, patient has:

- No loneliness

- Had complaints of loneliness in the past 12 months

- Unknown

\section{Social network, patient has:}

- Sufficient and strong social network

- Large but weak social network

- Small but strong social network

- Small and weak or no social network

- Unknown

11. Depressive complaints, patient has:

- No depressive complaints

- Depressive complaints

- Unknown

12. Anxiety complaints, patient has:

- No anxiety complaints

- Anxiety complaints

- Unknown

13. Somatoform complaints, patient has:

- No somatoform complaints

- Somatoform complaints

- Unknown

14. Other psychiatric complaints, patient has:

- No other psychiatric complaints

- Other psychiatric complaints

- Unknown

You went through all the domains that may have influence on the frailty status of the patient. Based on your prior knowledge of the patient, do you think this patient is frail?

- The patient is not frail

- The patient is frail

- The frailty status of the patient is unclear pilot study with two non-academic GPs. They randomly selected 10 patients aged $\geq 70$ years and older and screened them with the first version of the Easycare-TOS. They reported several points for improvement based on their screening experiences.

\section{Pilot study 2}

A more extensive feasibility test of the second version was carried out in seven academic GP practices situated in urban ( $n=1)$, suburban ( $n=4)$, and countryside $(n=2)$ areas. The GPs working in these practices randomly selected approximately 20 patients aged $\geq 70$ years. These patients were screened using the Easycare-TOS. In total, 141 patients were included. All participating professionals received a short written instruction on how to work with the Easycare-TOS. After screening the 20 patients, the participating professionals completed a questionnaire about their experiences with the Easycare-TOS. They were asked to report the time required for the procedure, whether they used the Easycare-TOS as described in the instruction, what barriers and facilitators they experienced for a successful implementation of the Easycare-TOS in GP practice, and how patients reacted to the Easycare-TOS. Sixteen professionals completed the questionnaire (seven primary care nurses and nine GPs).

After discussing the results of the second pilot study in the research group, a final version of the Easycare-TOS was produced.

In addition, the patient data obtained from pilot 2 were studied, in order to gain insight into how professionals decided whether a patient was frail or not frail. First, the outcomes of the frailty instrument will be described as percentages of frail and not frail patients. Secondly, the characteristics that determined whether a patient was more or less frail in the opinion of primary care professionals will be described. For this purpose, the data from the first step of the Easycare-TOS were analysed, as this questionnaire is the only one that was completed for all patients (the frail and the not frail).

\section{RESULTS}

Pilot studies: feasibility and adaptations

Pilot 1. The two GPs who participated in this pilot concluded that there was a difference in the complexity of the care context between older people, even within the group of frail people. After discussion with the GPs, the planning group concluded that specifically the frail patients with a complex care context are the patients in need of 


\section{Box 2. Examples of questions of two domains of the second step of the Easycare-TOS}

\author{
6. Seeing, hearing and communicating \\ 6.1. Can you see (with glasses if worn)? \\ - Yes \\ - With difficulty \\ - Cannot see at all \\ 6.2. Can you hear (with hearing aid if worn)? \\ - Yes \\ - With difficulty \\ - Cannot hear at all
}

6.3. Do you have difficulty in making yourself understood because of problems with your speech? - No difficulty

- Difficulty with some people

- Considerable difficulty with everybody

6.4. Can you use the telephone?

- Without help including looking up numbers and dialling

- With some help

- Unable to use the telephone

\section{Loneliness/social network}

10.1. Do you live alone?

- No

- Yes

10.2. Is there anyone who would be able to help you in case of illness or emergency?

- No

- Yes

10.3. Do you have contact with people in your neighbourhood?

- With few people, little contact

- With few people, but sufficient contact

- With many people, little contact

- With enough people, sufficient contact

10.4. Do you feel lonely?

- Never

- Sometimes

- Often integrated care. A complex care context represents the experienced problems with the organisation and coordination of care for a patient, for example, uncertainty about the treatment, unpredictability of the further course of the disease/problem, and disagreement about the treatment between caregivers or between a caregiver and the patient. ${ }^{14,21-26}$

Accordingly, it was decided to make a subdivision in the group of frail patients into: (1) frail without a complex care context; (2) frail with a complex care context. This subdivision is made after the assessment of the second step of the Easycare-TOS.

Pilot 2: time investment. The first step by the GP took a mean of 6.5 minutes per patient (range 3-10 minutes); the assessment of the second step by a primary care nurse took a mean of 57 minutes per patient (range 45-90 minutes); and the discussion of the assessment and final frailty decision between the GP and nurse took a mean of 8 minutes per patient (range 2-10 minutes). Eleven professionals (six GPs and five primary care nurses) (69\%) reported that the time investments had been worthwhile. Two (one GP and one primary care nurse) considered the method too time consuming and three did not answer this question.

Pilot 2: reaction of professionals to the Easycare-TOS. Six out of seven GP practices decided to work with the Easycare-TOS in the future. Fourteen professionals (88\%) thought that the Easycare-TOS would be an effective method for the identification of frail older patients. Two professionals did not answer this question.

\section{Box 3. Perceived barriers and facilitators for the implementation of the Easycare-TOS mentioned by professionals of pilot 2}

\begin{tabular}{ll} 
Barriers & Facilitators \\
\hline Professionals' appraisal & Professionals' appraisal \\
\hline $\begin{array}{l}\text { Assessment not resulting in a quantitative } \\
\text { cut-off score }\end{array}$ & $\begin{array}{l}\text { Assessment not resulting in a quantitative cut-off } \\
\text { score }\end{array}$ \\
\hline $\begin{array}{l}\text { Not incorporated in the routine of } \\
\text { daily practice }\end{array}$ & $\begin{array}{l}\text { Becoming aware of the seriousness of the situation } \\
\text { of some frail older patients }\end{array}$ \\
\hline $\begin{array}{l}\text { Organising home visit for assessment in } \\
\text { the second step is time consuming }\end{array}$ & $\begin{array}{l}\text { Provides starting points for further intervention } \\
\text { An implementation protocol is missing }\end{array}$ \\
$\begin{array}{ll}\text { Normally you only focus on domains where there are } \\
\text { problems, but now you have to check out all } \\
\text { domains of functioning }\end{array}$ \\
\hline $\begin{array}{l}\text { Contacting an informal caregiver is difficult } \\
\text { Patients may give socially desirable answers }\end{array}$ & It makes the GP aware of the diversity of problems \\
\hline $\begin{array}{l}\text { Too little experience with frailty in older } \\
\text { people; therefore it is hard to make the }\end{array}$ & It enhances patient satisfaction \\
final judgement on frailty &
\end{tabular}

\section{Pilot 2: reaction of patients.}

None of the professionals reported negative reactions of patients, and, according to 13 professionals (81\%), the reactions of patients were mainly positive.

Box 3 shows the barriers and facilitators for implementation of the Easycare-TOS reported by the professionals.

The professionals who worked with the Easycare-TOS in the second pilot reported that they had some difficulties making the final decision. Despite articulating an implicit understanding of the concept of frailty, most agreed that they had too little knowledge about frailty and a complex care context in older people to make a reliable final decision. Therefore, it was decided to develop an educational programme, with the aim to give the professionals sufficient background information about frailty and a complex care context. This programme 


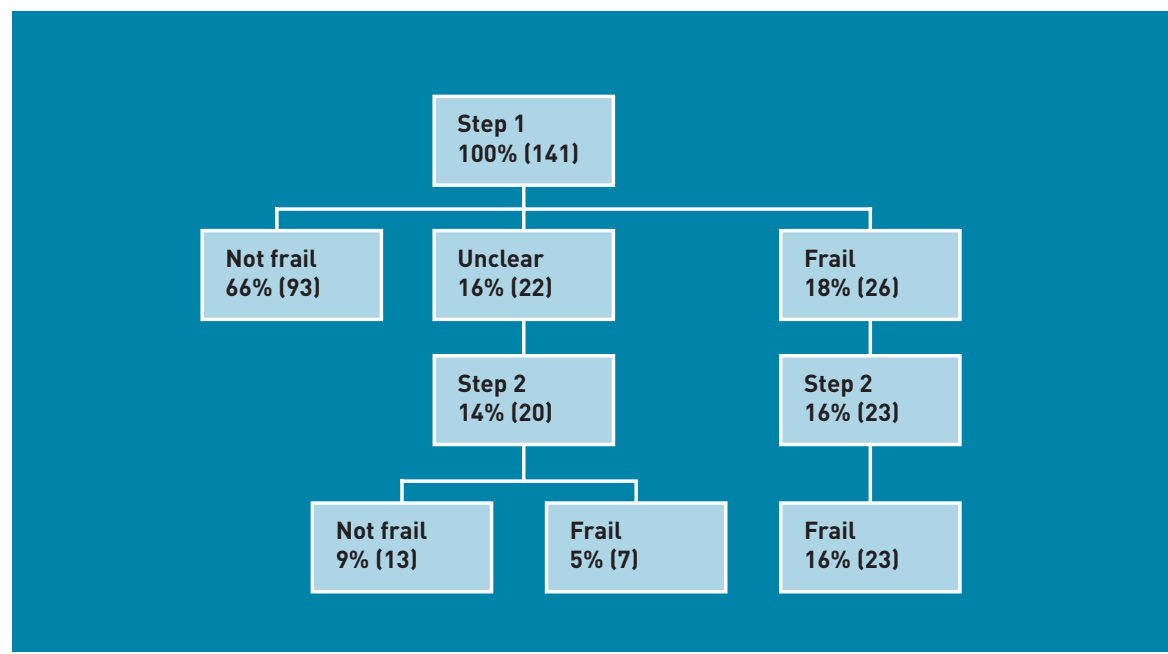

Figure 1. The distribution of patients over the different groups of the subsequent identification steps of pilot 2.

\section{Funding}

This project was funded by grant 60-61900-98217 of the Dutch National Care for the Elderly Programme, coordinated and sponsored by ZonMw, The Netherlands Organisation for Health Research and Development.

\section{Ethical approval}

Because of the nature of the study, the local ethics committee declared that no formal approval was required for the pilot studies.

\section{Provenance}

Freely submitted; externally peer reviewed.

\section{Competing interests}

The authors have declared no competing interests.

\section{Discuss this article}

Contribute and read comments about this article on the Discussion Forum: http://www.rcgp.org.uk/bjgp-discuss also trains the professionals in using the Easycare-TOS

\section{Pilot studies: the frailty decision}

In pilot 2, 141 patients were included in the first step of the Easycare-TOS (mean age 77 years [standard deviation $=6$ years] and $62 \%$ female). The prevalence of frailty in this study population is $24 \%$. Two of the frail patients were categorised as having a complex care context. The distribution of the patients over the different groups (not frail, frail, unclear) in the subsequent identification steps is displayed in Figure 1.

After analysing the data from the several questions of step 1, it can be concluded that, with the exception of dementia, there were no other single factors that always classified older people as 'frail'. However, of the 21 patients who were highly dependent on professional or informal care for activities of daily living', 19 were classified as 'frail'. The other two patients were classified as 'not frail', because they had no other major problems and had a strong social network with a strong informal carer. Patients with an insufficient social network, or who had fallen multiple times in the preceding year, were never classified as 'not frail'. When 'activities of daily living' and 'mobility' were unknown by the GP, the patient was always classified as 'unclear', and never as 'frail' based on other characteristics.

\section{DISCUSSION}

\section{Summary}

This research developed a new method for the identification of communitydwelling frail older people as the target population for integrated care, the Easycare-TOS. This method has several innovative characteristics. First, it uses prior knowledge of the GP. Secondly, the professionals appraisal, taking tacit knowledge into account, is decisive in the frailty decision. In this way, the EasycareTOS makes optimal use of available information and the experience of the GP.

Interest in the identification of frailty is growing in primary care. In this study it was decided to use a two-step approach as proposed by De Lepeleire in 2009, with a simple heuristic tool as the first step, and a more complex assessment as the second. ${ }^{19}$ Several short instruments were suggested for use as the first step. ${ }^{20,27}$ These short instruments had characteristics that make them less suitable for the purpose of this study. First, most instruments are developed in the research realm and not tested in a primary care setting. ${ }^{7,28,29}$ Second, some instruments are impractical for primary care, for example, the screening test of Moore et al, and the Survey of Health, Ageing and Retirement in Europe - Frailty Instrument (SHARE-FI), which comprise several performance-based tests. ${ }^{28,30}$ Third, many of these short instruments are selfassessment instruments, for example the TFI, ${ }^{10}$ Groningen Frailty Instrument (GFI), ${ }^{31}$ Programme de Recherche sur l'Intégration des Services pour le Maintien de l'Autonomie (PRISMA-7), ${ }^{32}$ and the Sherbrooke postal questionnaire. ${ }^{33}$ Self-assessment instruments have the lowest response rate in the most vulnerable group of patients, and the reliability of the information from frail patients, especially in case of cognitive problems, is questionable. ${ }^{27}$ Therefore this study developed its own first step, specifically developed for use in primary care.

\section{Strengths and limitations of the study}

Most professionals who participated in the pilot studies reported that the identification of frailty with the Easycare-TOS had added value, and that the time investment was acceptable. Six out of seven GP practices wanted to work with the Easycare-TOS in the future. These results show that the study succeeded in developing an efficient, flexible, and feasible identification method.

However, this method also has some limitations. First, using the professionals appraisal as a frailty judgement, instead of a numerical score, increases the interobserver variability. With the educational programme, all professionals will be given the same background information on frailty and this will increase the comparability between the professionals.

A second limitation is the considerable time investment required for the 
assessment of the second step by a primary care nurse. Time constraints have been reported before as a barrier to the implementation of comparable interventions. $^{34}$ Therefore, a stepwise approach was used in the Easycare-TOS. After the first step, two-thirds of patients appeared to be 'not frail', which meant that a relatively large number of patients did not need further assessment; this is already a significant improvement in efficiency.

\section{Comparison with existing literature}

The study aim was to identify frail older people for the purpose of identifying a target population for integrated care. This is not a new concept; Wilhelmson et al stated that frail older people are in need of integrated care. They proposed geriatric screening and multidimensional assessment as a way to enhance integration. ${ }^{3}$ De Lepeleire et al stated that frailty provides a conceptual basis for a health-based integrative approach. ${ }^{19}$ With the development of the Easycare-TOS, the present researchers wanted to provide a good starting point for providing integrated care. Use of the Easycare-TOS enables the GP not only to identify the frail patients in his population, but also to have a first assessment of the problems of the frail patients. This makes the Easycare-TOS a good starting point for providing proactive care and individualised multidisciplinary care planning, which is the first step in providing integrated care. The next step should be that the professionals of the different disciplines enhance collaboration in order to provide individualised and multidisciplinairy care. ${ }^{35,36}$

\section{Implications for practice and research}

From the results of the pilot studies, it can be concluded that the EasycareTOS meets the predefined efficiency, flexibility, and acceptability requirements. The Easycare-TOS is suitable for use in primary care. Follow-up prospective data will be required to show whether the Easycare-TOS also identifies the group of older patients who will benefit most from integrated care. 


\section{REFERENCES}

1. Boeckxstaens P, De Graaf P. Primary care and care for older persons: position paper of the European Forum for Primary Care. Qual Prim Care 2011; 19(6): 369-389.

2. Erler A, Bodenheimer T, Baker R, et al. Preparing primary care for the future - perspectives from the Netherlands, England, and USA. Z Evid, Fortbild Qual Gesundheits 2011; 105(8): 571-580.

3. Wilhelmson $\mathrm{K}$, Duner A, Eklund $\mathrm{K}$, et al. Design of a randomized controlled study of a multi-professional and multidimensional intervention targeting frail elderly people. BMC Geriatr 2011; 11: 24.

4. Kodner DL. The quest for integrated systems of care for frail older persons. Aging Clin Exp Res 2002; 14(4): 307-313.

5. Singer SJ, Burgers J, Friedberg M, et al. Defining and measuring integrated patient care: promoting the next frontier in health care delivery. Med Care Res Rev 2011; 68(1): 112-127.

6. Clarkson P, Brand C, Hughes J, Challis D. Integrating assessments of older people: examining evidence and impact from a randomised controlled trial. Age Ageing 2011; 40(3): 388-391.

7. Lacas A, Rockwood K. Frailty in primary care: a review of its conceptualization and implications for practice. BMC Med 2012; 10: 4

8. Bandeen-Roche K, Xue QL, Ferrucci L, et al. Phenotype of frailty: characterization in the women's health and aging studies. J Gerontol A Biol Sci Med Sci2006; 61(3): 262-266.

9. Fried LP, Tangen CM, Walston J, et al. Frailty in older adults: evidence for a phenotype. J Gerontol A Biol Sci Med Sci2001; 56(3): M146-156.

10. Gobbens RJ, van Assen MA, Luijkx KG, et al. The Tilburg Frailty Indicator: psychometric properties. J Am Med Dir Assoc 2010; 11(5): 344-355.

11. Jones D, Song X, Mitnitski A, Rockwood K. Evaluation of a frailty index based on a comprehensive geriatric assessment in a population based study of elderly Canadians. Aging Clin Exp Res 2005; 17(6): 465-471.

12. Rockwood K, Mitnitski A. Frailty in relation to the accumulation of deficits. J Gerontol A Biol Sci Med Sci2007; 62(7): 722-727.

13. Kienle GS, Kiene H. Clinical judgement and the medical profession. J Eval Clin Pract 2011; 17(4): 621-627.

14. de Jonge P, Huyse FJ, Stiefel FC. Case and care complexity in the medically ill. Med Clin North Am 2006; 90(4): 679-692.

15. Richardson J. The Easy-Care assessment system and its appropriateness for older people. Nurs Older People 2001; 13(7): 17-19.

16. Gobbens RJ, Luijkx KG, Wijnen-Sponselee MT, Schols JM. Toward a conceptual definition of frail community dwelling older people. Nurs Outlook 2010; 58(2): $76-86$.

17. Strawbridge WJ, Shema SJ, Balfour JL, et al. Antecedents of frailty over three decades in an older cohort. J Gerontol B Psychol Sci Soc Sci 1998; 53(1): S9-16.

18. Rockwood K, Hogan DB, MacKnight C. Conceptualisation and measurement of frailty in elderly people. Drugs Aging 2000; 17(4): 295-302.
19. De Lepeleire J, lliffe S, Mann E, Degryse JM. Frailty: an emerging concept for general practice. Br J Gen Pract 2009; 59(562): 364-369.

20. Pialoux T, Goyard J, Lesourd B. Screening tools for frailty in primary health care a systematic review. Geriatr Gerontol Int 2012; 12(2): 189-197.

21. de Jonge P. Huyse FJ, Slaets JP, et al. Care complexity in the general hospital: results from a European study. Psychosomatics 2001; 42(3): 204-212.

22. Kelleher $\mathrm{C}$. Relationship of physician ratings of severity of illness and difficulty of clinical management to length of stay. Health Serv Res 1993; 27(6): 841-855.

23. Holden LM. Complex adaptive systems: concept analysis. J Adv Nurs 2005; 52(6): 651-657

24. Plsek PE, Wilson T. Complexity, leadership, and management in healthcare organisations. BMJ 2001; 323(7315): 746-749.

25. Olde Rikkert MG, Schers HJ, Melis RJ. Defining patient complexity. Ann Intern Med 2012; 156(8): 606; author reply 607.

26. Grant RW, Ashburner JM, Hong CC, et al. Defining patient complexity from the primary care physician's perspective: a cohort study. Ann Intern Med 2011: 155(12): 797-804.

27. Daniels R, van Rossum E, Beurskens A, et al. The predictive validity of three selfreport screening instruments for identifying frail older people in the community. BMC Public Health 2012; 12: 69

28. Romero-Ortuno R, Walsh CD, Lawlor BA, Kenny RA. A frailty instrument for primary care: findings from the Survey of Health, Ageing and Retirement in Europe (SHARE). BMC Geriatr 2010; 10: 57.

29. Saliba D, Elliott M, Rubenstein LZ, et al. The Vulnerable Elders Survey: a tool for identifying vulnerable older people in the community. J Am Geriatr Soc 2001 49(12): 1691-1699.

30. Moore AA, Siu AL. Screening for common problems in ambulatory elderly: clinical confirmation of a screening instrument. Am J Med 1996; 100(4): 438443

31. Steverink N, Slaets JPJ, Schuurmans $H$, van Lis M. Measuring frailty: developing and testing the GFI (Groningen Frailty Indicator). Gerontologist 2001; 41: 236237.

32. Raiche M, Hebert R, Dubois MF. PRISMA-7: a case-finding tool to identify older adults with moderate to severe disabilities. Arch Gerontol Geriat 2008; 47(1): 9-18.

33. Hebert R, Bravo G, KornerBitensky N, Voyer L. Predictive validity of a postal questionnaire for screening community-dwelling elderly individuals at risk of functional decline. Age Ageing 1996; 25(2): 159-167.

34. van Eijken $M$, Melis R, Wensing M, et al. Feasibility of a new community-based geriatric intervention programme: an exploration of experiences of GPs, nurses, geriatricians, patients and caregivers. Disabil Rehabil 2008; 30(9): 696-708.

35. Eklund K, Wilhelmson K. Outcomes of coordinated and integrated interventions targeting frail elderly people: a systematic review of randomised controlled trials. Health Soc Care Community 2009; 17(5): 447-458.

36. Rickenbach MA, Wedderburn C. Follow the yellow brick road: integrated care can we do better? Br J Gen Pract 2012; 62(601): 587-589. 